Published in final edited form as:

Cognition. 2019 April ; 185: 71-82. doi:10.1016/j.cognition.2019.01.007.

\title{
Conscious and unconscious memory differentially impact attention: Eye movements, visual search, and recognition processes
}

\author{
Michelle M. Rameya,b,c, ${ }^{\star}$, Andrew P. Yonelinas ${ }^{a, b}$, John M. Henderson ${ }^{a, c}$ \\ aDepartment of Psychology, University of California, Davis, CA, USA \\ ${ }^{b}$ Center for Neuroscience, University of California, Davis, CA, USA \\ ${ }^{c}$ Center for Mind and Brain, University of California, Davis, CA, USA
}

\begin{abstract}
A hotly debated question is whether memory influences attention through conscious or unconscious processes. To address this controversy, we measured eye movements while participants searched repeated real-world scenes for embedded targets, and we assessed memory for each scene using confidence-based methods to isolate different states of subjective memory awareness. We found that memory-informed eye movements during visual search were predicted both by conscious recollection, which led to a highly precise first eye movement toward the remembered location, and by unconscious memory, which increased search efficiency by gradually directing the eyes toward the target throughout the search trial. In contrast, these eye movement measures were not influenced by familiarity-based memory (i.e., changes in subjective reports of memory strength). The results indicate that conscious recollection and unconscious memory can each play distinct and complementary roles in guiding attention to facilitate efficient extraction of visual information.
\end{abstract}

\section{Keywords}

Recognition; Contextual cueing; Visual search; Eyetracking; Memory; Implicit memory

\section{Introduction}

How people move their eyes can provide important clues about the contents of their mind. For example, how the eyes move while viewing an object or scene can differ for previously encountered items compared to those that are novel, suggesting that eye movements can be

*Corresponding author at: 1 Shields Ave, Davis, CA 95618, USA. mmramey@ ucdavis.edu (M.M. Ramey). Author contributions

Conceptualization, Methodology, Writing - Review \& Editing, J.M.H., A.P.Y., M.M.R.; Funding acquisition, A.P.Y., J.M.H.; Formal

Analysis, Investigation, Writing - Original Draft, Visualization, M.M.R.

Supplementary material

Data used for analyses are available through the Open Science Framework: https://osf.io/su5pa/forbrevity.

Conflict of interest

Authors declare no conflict of interest. 
used to reveal memory for prior experiences (Althoff \& Cohen, 1999; Hannula, 2010; Henderson \& Hollingworth, 2003; Hollingworth, Williams, \& Henderson, 2001; Kafkas \& Montaldi, 2012; Ryan, Hannula, \& Cohen, 2007). Interestingly, there is evidence that eye movements may reveal influences of memory even when participants have no conscious awareness of that memory (Hannula, 2010; Hannula, Baym, Warren, \& Cohen, 2012; Ryan, Althoff, Whitlow, \& Cohen, 2000; Smith \& Squire, 2017). Whether these effects are truly due to conscious or unconscious memory, however, is hotly contested, because other studies have found that eye movement differences between new and previously viewed items are limited to trials in which participants are aware of their memory for the items (Smith, Hopkins, \& Squire, 2006; Smith \& Squire, 2008).

The debate about conscious and unconscious influences on visual attention has been particularly lively in the literature on contextual cueing — a paradigm in which participants search for a target letter in an array of distractors (Chun \& Jiang, 1998). Repetition of the array and target combination leads to performance improvements due to memory for the context (i.e., the array of distractors), as evidenced by a decrease in response time, a more accurate first eye movement toward the target location, and a decrease in the number of eye movements needed to reach the target (Peterson \& Kramer, 2001). Improved search performance in this task is often attributed to unconscious memory, because when participants are subsequently asked if they recognize the studied arrays, they report little or no memory for those arrays (Chun, 2000; Chun \& Jiang, 1998; Chun \& Phelps, 1999; Johnson, Woodman, Braun, \& Luck, 2007). However, because these recognition tests did not probe memory confidence and are typically based on small numbers of trials, the failure to find a relationship with conscious memory could be attributed to imprecise memory probes and insufficient power (Goujon, Didierjean, \& Marmèche, 2007; Schlagbauer, Muller, Zehetleitner, \& Geyer, 2012; Smyth \& Shanks, 2008). Moreover, in contextual cueing studies using realistic scenes as contexts, participants report recognizing the scenes well above chance after the conclusion of the study (Brockmole \& Henderson, 2006a, 2006b). It is therefore currently unknown whether the improvements in search performance from contextual cueing are due to conscious memory, or to unconscious memory. Furthermore, it is not known whether conscious and unconscious memory might be able to influence eye movements in different ways.

\subsection{Current research}

In the present experiment, we investigated these possibilities by combining a contextual cueing paradigm with a confidence-based recognition method to isolate different states of subjective memory awareness, and used eyetracking to examine different patterns of eye movements during search. Participants first completed a learning phase where they searched realistic scenes for embedded target letters, indicating when they identified the letter (i.e., either a "T" or an "L"). During the subsequent test phase (see Fig. 1), participants were presented with a mixture of old scenes (i.e., scenes that had been encountered in the earlier learning phase) and new scenes; for each scene, they first made a recognition judgment, and then searched for the target letter as they did in the learning phase. Memory awareness was measured by asking participants to rate memory confidence for each scene on a 6-point scale during the recognition judgment. For each of these test scenes, participants were told that if 
they could consciously recollect some qualitative aspect of the initial learning event, such as what they thought about when the scene was encountered earlier, they should respond "Recollect old (6);" otherwise, they rated their memory confidence by responding "I'm sure it's old (5)," “Maybe it's old (4)," “I don't know (3)," "Maybe it's new (2)," or “I'm sure it's new (1)."

To isolate eye movement patterns related to unambiguously unconscious memory, we examined the scenes that participants were confident had not been studied (i.e., receiving a response of "I'm sure it's new"), and in this way we excluded any scenes for which there was even a weak sense of conscious memory (i.e., the "maybe it's old', "I don't know" and "maybe it's new" trials). Conversely, to assess eye movement patterns that were related to unambiguously conscious memory, we examined the old scenes that were confidently recognized as studied and for which participants reported being able to retrieve specific details about the study event (i.e., "recollect old"). We also examined intermediate levels of memory confidence to determine if memory strength might be related to eye movement patterns in a manner similar to conscious or unconscious memory. There is evidence from behavioral and neural studies that intermediate-confidence recognition responses may rely on a familiarity process that is distinct from conscious recollection (e.g., Eichenbaum, Yonelinas, \& Ranganath, 2007; Yonelinas, 2002, but also see Donaldson, 1996), but whether they are related to distinct types of eye movements is not yet clear (e.g., Kafkas \& Montaldi, 2011, 2012; Sharot, Davidson, Carson, \& Phelps, 2008).

The influences of the above memory processes on contextual cueing performance were assessed in the present study using two eye movement measures, which have been shown to be sensitive to contextual cueing effects and are reflective of eye movement behavior at distinct points in the search process. To assess search-relevant behavior early in the trial, we measured the degree error of the first eye movement in a trial (i.e., first saccade accuracy) in terms of whether it was aimed toward the target, similar to the measure used by Peterson and Kramer (2001). To assess search processes that take place throughout the course of the trial, we measured scanpath efficiency — the efficiency of the overall search path—by dividing the total distance traveled by the shortest possible path to the target (Castelhano \& Henderson, 2007). We expected that both of these eye movement measures of performance would be improved for scenes that were repeated compared to scenes that were new, and would thus reflect influences of memory.

Given the ambiguity of the existing literature regarding the role of conscious and unconscious memory in eye movement guidance, we did not have strong a priori predictions about how the eye movement measures would be related to reports of memory awareness. However, a number of different outcomes would be of theoretical interest. For example, the findings may show that eye movements are related selectively to either conscious or unconscious memory, which would be useful in resolving the debate about whether eye movements reflect conscious or unconscious memory processes. Conversely, both conscious and unconscious memory may influence each of the eye movement measures, suggesting that the influence of memory on eye movements may not clearly dissociate along the lines of conscious awareness. Finally, conscious and unconscious memory may be related to distinct 
types of eye movements, suggesting that conscious and unconscious memory processes may contribute to visual search in distinct ways.

\section{Materials and methods}

\subsection{Participants}

Twenty-six undergraduate students were recruited from the participant pool at the University of California, Davis. Three participants did not meet criteria for high-quality eyetracking (i.e., at least $75 \%$ signal, such that the eyetracker was able to record the position of the eyes at least $75 \%$ of the time) and were excluded from analysis. Signal in the 23 participants included in the analyses was $93 \%$ on average. The included sample exceeded the sample size needed to detect the weakest effects previously reported in similar studies with $95 \%$ power. All participants had normal or corrected-to-normal vision and participated in exchange for course credit. Informed consent was obtained from all participants.

\subsection{Stimuli}

Stimuli consisted of 160 digital photographs of real-world indoor and outdoor scenes (Fig. 1). Each scene contained a small grey " $L$ " or " $T$ " as the search target. The search targets were randomly placed using a custom MATLAB script to ensure an even distribution of target locations across scenes, with the center region $(160 \times 160 \mathrm{px})$ and periphery of the scenes (outer 15\%) excluded as possible target locations. Scenes were subsequently manually inspected, and if targets were not visible, they were moved to the nearest possible point at which they were visible. This procedure resulted in targets with $\mathrm{x}$-coordinates ranging from $154 \mathrm{px}$ to $871 \mathrm{px}$, and y-coordinates ranging from $115 \mathrm{px}$ to $653 \mathrm{px}$. The initial scene set consisted of 300 scenes, and these scenes were subsequently normed for search difficulty; the final set of 160 scenes was produced by eliminating the easiest and most difficult scenes. For a given scene, the identity of the target letter was consistent and appeared in the same location across presentations.

Of the 160 scenes, 64 scenes were presented once in the learning phase (i.e., $1 \times$ scenes), 64 scenes were presented three times in the learning phase (i.e., $3 \times$ scenes), and 32 scenes served as lures in the test phase (i.e., new scenes). To minimize any stimulus effects, two counterbalances were used: assignment of scene to condition was counterbalanced such that each scene appeared in two of these three possible conditions $(1 \times, 3 \times$, new) across participants. That is, across participants, each scene appeared as a $1 \times$ and new scene, a $1 \times$ and $3 \times$ scene, or a $3 \times$ and new scene.

\subsection{Apparatus}

Participants sat $85 \mathrm{~cm}$ away from the screen, such that scenes spanned approximately $25^{\circ} \times$ $19^{\circ}$ of visual angle at a resolution of $1024 \times 768$ pixels. Targets spanned approximately $0.27^{\circ} \times 0.27^{\circ}$ of visual angle. Eye movements were recorded using an SR research Eyelink 1000+ tower mount eyetracker, which sampled at $1000 \mathrm{~Hz}$. Eye movements were measured from the right eye, although vision was binocular, and a chin and forehead rest were used to prevent head movements. Participants' eye movements were tracked throughout the experiment. 


\subsection{Procedure}

The first half of the experiment consisted of a learning phase, during which participants searched a series of scenes for their targets. Each scene was preceded by a fixation cross to ensure that participants were looking at the center of the screen upon scene presentation. Participants were asked to find the search target in each scene and use the keyboard to indicate whether it was an "L" or a "T". Each trial was terminated at response, or after $20 \mathrm{~s}$ without response. Participants were not aware that there would be a subsequent memory test and were not told that scenes would be repeated.

The learning phase was comprised of 128 unique scenes, with 64 scenes presented once (i.e. $1 \times$ scenes) and 64 scenes presented three times (i.e. $3 \times$ scenes), for a total of 256 trials. The $3 \times$ scenes were included to assess contextual cueing effects during the learning phase over repeated searches. Based on prior research, we anticipated near-ceiling memory performance for the $3 \times$ scenes, and therefore included the $1 \times$ scenes to generate a more even distribution of recognition responses for the purposes of test phase analyses by memory type. The scenes were randomly ordered throughout the learning phase with the constraint that at least 3 different scenes were interleaved between repeated presentations of a given scene. The participants were given short breaks every 50 trials and between the learning and test phases, and the eyetracker was re-calibrated after each break to prevent drift. The delay between the end of the learning phase and the start of the test phase instructions was approximately three minutes.

In the test phase (Fig. 1), each trial started with a fixation cross, followed by a $400 \mathrm{~ms}$ preview of a scene without its target; the scene was either one that had been presented in the learning phase, or a new scene. Each preview was then replaced by a memory response screen. Participants were given as long as they needed to provide a memory response indicating whether or not they recognized the scene from the learning phase. Response options fell on a 1-5 and recollect scale (Yonelinas, 2002) made up of "sure new", "maybe new", "don't know", "maybe old", "sure old", and "recollect old". Participants were instructed and tested on how to use this scale prior to beginning the test phase. A response of "recollect old" indicated that a participant could recall details of their experience of having seen the image in the learning phase. Examples given to participants included remembering an emotion they felt during prior exposure to the scene, and remembering ambient noise or sensations experienced while previously viewing the scene. The other responses fell on a continuous gradient ranging from no memory to strong memory for a scene. Importantly, participants were instructed that a response of "sure old" indicated high memory confidence comparable to that of "recollect old" responses, but without the additional episodic details.

After the memory probe in each trial, participants were shown another fixation cross followed by the same scene- this time with the search target included — and searched as they did in the learning phase. The test phase consisted of 160 trials (128 old scenes and 32 lures), with one trial for each unique scene. 


\subsection{Analysis}

Statistical analyses were conducted using linear mixed effects models with crossed random effects of participant and image, which allowed us to harness trial-by-trial (i.e., withinsubjects) data while controlling for individual differences and stimulus effects. The models were estimated using the lmerTest package in $\mathrm{R}$ (Kuznetsova, Brockhoff, \& Christensen, 2017), and were fit using maximum likelihood. The degrees of freedom, estimated using the Satterthwaite approximation, and $t$ values used were output by the linear mixed effects model for the variables of interest. Effect sizes were calculated as classical Cohen's $d$, as $\frac{2 t}{\sqrt{d f}}$ (Rosenthal \& Rosnow, 1991). Trials with less than $75 \%$ signal were excluded from analysis (5\% of trials).

The models for each analysis of the effects of memory on eye movements (Section 3.2) were specified by regressing the eye movement measure in question (i.e., first saccade accuracy, scanpath ratio, or delta distance) on a memory variable, which depended on the type of memory being assessed: conscious recollection, unconscious memory, or familiarity strength (see Table 1). In order to plot the data in a way that most directly reflects the analyses, Figs. 2, 3a, and $4 \mathrm{a}$ are derived from the linear mixed effects models. As such, the plotted means are estimated marginal means, and the error bars represent the standard error of the estimated marginal means, both derived from the models. This allows for both within- and between-subjects variance to be taken into account, and controls for participant and stimulus effects. (However, the plots are similar when the raw data is plotted. See Appendix A for more details.)

\section{Results}

\subsection{Contextual cueing effects}

Search speed.-The learning phase included a mixture of scenes that were presented once, which we used to examine memory in the subsequent test phase, and scenes that were presented three times, which allowed us to examine contextual cueing effects prior to probing recognition memory. For the scenes that were presented three times in the learning phase, participants showed a significant increase in search speed across repeated presentations, $t(6410)=-16.84, p<.0001, d=-0.42$ (first presentation $\mathrm{M}=6030 \mathrm{~ms}$, third presentation $\mathrm{M}=3296 \mathrm{~ms}$ ), and repeated scenes had faster search speed than new scenes in the test phase, $t(2347)=-9.44, p<.0001, d=-0.39$, demonstrating the standard contextual cueing effect (Brockmole \& Henderson, 2006a, 2006b).

Eye movements.-To investigate the behavior of the first eye movement made in the search process, first saccade accuracy was calculated as the degree error between the vector defined by the first saccade and the vector defined by the ideal path to the target from the central fixation cross (Table 2). Lower values indicate lower degree error and therefore better performance, such that the first eye movement was aimed more directly towards the target. First saccade accuracy improved across presentations in the learning phase (across presentations 1-3), $t(3834)=-2.37, p=.018, d=-0.08$, and throughout the experiment as a whole (across presentations 1-4), $t(4971)=-7.88, p<.0001, d=-0.22$ (Fig. 2a). 
To investigate eye movement behavior throughout the search period, we quantified scanpath efficiency over the course of the trial as the scanpath ratio (Castelhano \& Henderson, 2007), the ratio of the observed scanpath to the most direct possible path to the target (Table 2). Specifically, we defined scanpath ratio as the total distance travelled by the eyes in the course of finding the target (calculated by summing the length of saccades) divided by the length of the direct path from the starting point to the target. As such, a scanpath ratio of 1 indicates ideal performance, such that the path taken by the eyes was the same length as the ideal path, whereas higher scanpath ratio values indicate less efficient paths. Scanpath ratio also improved over the course of the learning phase, $t(3379)=-14.73, p<.0001, d=-0.51$, and throughout the entire experiment, $t(4317)=-18.65, p<.0001, d=-0.57$ (Fig. 2b).

Both first saccade accuracy and scanpath efficiency were also significantly better for repeated scenes than new scenes in the test phase (first saccade accuracy: $t(1515)=-5.87, p$ $<.0001, d=-0.30$; scanpath efficiency: $t(2131)=-9.37, p<.0001, d=-0.41)$. Together, these analyses suggest that the two eye movement measures improved as a result of contextual cueing, and that these effects were observed even prior to the test phase in which recognition judgments were required.

\subsection{Behavior during the test phase}

Memory accuracy.-The percentage of scenes receiving a recognition confidence response corresponding to "recollect," "sure old," "maybe old," "don't know," "maybe new," and "sure new," respectively, were $41 \%, 34 \%, 10 \%, 6 \%, 5 \%, 4 \%$, for scenes that were presented three times during the learning phase; $11 \%, 24 \%, 14 \%, 12 \%, 19 \%, 20 \%$ for scenes presented once during the learning phase; and $0.5 \%, 2.5 \%, 5 \%, 13 \%, 25 \%, 54 \%$ for new scenes. These results indicate that participants recognized more of the old than new scenes, and they used the full range of response confidence ratings. Only the scenes presented once in the learning phase and the new scenes were used in each of the following test phase analyses.

First saccade accuracy.-Fig. 3a presents test phase first saccade accuracy, in terms of degree error, as a function of memory response and type of scene (i.e., old versus new). To determine whether first saccade accuracy was influenced by recollection, we examined the test phase eye movement data from old scenes that were endorsed as "recollect old" (i.e., recollection for the scene) and compared this to the eye movement data from old scenes endorsed as "sure old" (i.e., high-confidence familiarity with the scene) (Table 1). We found that conscious recollection of a scene drove the first saccade more directly toward the target than did high-confidence familiarity with a scene, $t(327)=-3.6, p<.001, d=-0.40$. This result indicates that conscious recollection improved the accuracy of the first eye movement, such that the first saccade was more likely to be aimed toward the target in scenes that were recollected.

To determine whether first saccade accuracy was influenced by unconscious memory, we examined the test phase data from old scenes that were endorsed as "sure new" and compared them to the data from new scenes that were endorsed as "sure new" (Table 1). This strict criterion for unconscious memory (i.e., only considering scenes that were 
endorsed as "sure new" rather than all misses) ensured that none of the scenes used in the unconscious memory contrast were contaminated by conscious recollection or familiarity, and that the scenes differed only in terms of whether or not the participant had seen them previously. Put differently, we compared first saccade accuracy between high-confidence misses and high-confidence correct rejections. First saccade accuracy did not differ between the new scenes and the old scenes that participants were confident they had not seen before, $t$ $(264)=-1.26, p=.21, d=-0.16$, indicating that unconscious memory did not significantly improve first saccade accuracy. Additionally, Bayesian analysis provided substantial evidence for the null hypothesis, suggesting that the numerically better first saccade accuracy for previously viewed scenes was almost five times more likely to be explained by chance than by unconscious memory $\left(\mathrm{BF}_{10}=0.22\right) .{ }^{1}$

Lastly, to determine whether first saccade accuracy was influenced by familiarity strength, we examined the test phase data for old scenes that were not endorsed as "recollect old," and assessed whether there was a linear relationship between familiarity confidence and first saccade accuracy (Table 1). That is, first saccade accuracy was compared across the gradient of responses ranging from "sure new" to "sure old" (denoted by the regression line over the gray points in Fig. 3a). First saccade accuracy did not differ across memory strength, $\beta=$ $0.02, t(633)=0.63, p=.54$, indicating that it was not affected by familiarity, with substantial evidence for the null hypothesis $\left(\mathrm{BF}_{10}=0.11\right)$.

To further characterize the relationship between first saccade accuracy and memory, we examined the density plot (i.e., smoothed histogram) of the distributions of first saccade accuracy by memory type (Fig. 3b). The figure shows that recollected scenes were associated with a large proportion of very accurate trials, such that the first saccades in these trials were within approximately 60 degrees of the direct path to the target. For the recollected scenes, the trials that did not have these highly accurate first saccades appeared to have degree errors distributed roughly evenly across degree error values greater than $60^{\circ}$, which may represent a distribution of guessing-based responses. That is, the distribution of degree error for recollected scenes appears visually analogous to a mixture model consisting of a highly precise distribution (i.e., $<60^{\circ}$ ), and a guessing distribution (Zhang \& Luck, 2008). This suggests that recollection may improve average first saccade accuracy by causing a subset of first saccades to be highly accurate, rather than causing a diffuse, more incremental improvement in first saccade accuracy across all recollected trials. In contrast, the distributions for old scenes that were not recollected and new scenes were similar to each other and generally inaccurate, leading to distributions spread across the full range of degree error values. This suggests that for scenes that were not recollected, first saccades were not systematically related to the target location, and therefore did not appear to be guided by memory.

Scanpath efficiency.-Fig. 4a presents test phase scanpath efficiency, quantified as scanpath ratio, as a function of memory response and type of scene. The same memory contrasts and scenes that were used for the analyses of first saccade accuracy were used for the analyses of scanpath ratio as well (Table 1). Within scenes given "sure new" responses,

\footnotetext{
${ }^{1}$ By convention, a BF $10<0.33$ indicates substantial evidence for the null hypothesis (Jeffreys, 1961).
} 
scanpath ratio was significantly lower for old scenes than for new scenes, $\mathrm{t}(331)=-3.76, p$ $<.001, d=-0.42$ - that is, scanpath ratio was lower for high-confidence misses than for high-confidence correct rejections. This indicates that unconscious memory for a scene led to a more efficient path to the target, even when participants were highly confident that they had not viewed the scene previously. In contrast, scanpath ratio was not improved by recollection compared to high-confidence familiarity, $t(307.4)=-1.47, p=.14, d=-0.17$, nor by familiarity strength overall, $\beta=-0.02, t(908)=-0.67, p=.51$.

When scanpath ratio was recalculated from the second saccade onward to provide complete temporal separation from first saccade accuracy (i.e., excluding the first saccade), the same pattern of results was obtained, such that only unconscious memory improved efficiency (unconscious memory: $t(375)=-2.28, p=.02, d=-0.24$; recollection: $t(361)=-0.91, p=$. $36, d=-0.10$; familiarity: $\beta=-0.03, t(1022)=-0.98, p=.33)$. Furthermore, Bayesian analysis provided substantial evidence for the null effect of both conscious recollection $\left(\mathrm{BF}_{10}=0.15\right)$ and familiarity $\left(\mathrm{BF}_{10}=0.14\right)$, such that in each case, the numerical differences in scanpath ratio were more than six times more likely to be explained by chance than by recollection or familiarity. Thus, memory-related increases in scanpath efficiency were not observed for conscious recollection or memory strength, but rather were limited to unconscious memory.

Although analysis of scanpath ratio indicated that unconscious memory increased the overall efficiency of the eyes in a trial, the underlying mechanism is unclear: it is equally plausible that the improvement in scanpath ratio was simply driven by a reduction in the total number of saccades made in a trial, or by another mechanism such as an enhancement of the efficiency of each individual saccade. A secondary analysis aimed at testing this showed that the effect of unconscious memory on scanpath ratio was not solely driven by a reduction in the number of saccades needed to reach the target. Specifically, the number of saccades made in a trial was only marginally reduced by unconscious memory, $t(371)=-1.90, p=.05$ (Fig. 4d). In contrast, when the number of saccades made in a trial was covaried in the model assessing the influence of unconscious memory on scanpath ratio, the effect of unconscious memory on scanpath ratio not only remained, but was in fact strengthened, $t(310)=-4.11, p<.0001, d=-0.47$. Therefore, unconscious memory appeared to guide the eyes such that each saccade was more efficient, rather than simply reducing the number of saccades needed to reach the target.

Decomposing scanpath efficiency.-The finding that scanpath ratio was significantly better for old scenes endorsed as "sure new" compared to new scenes endorsed as "sure new" indicates that unconscious influences of memory led to a more efficient scanpath. One interpretation of this effect is that the eyes are gradually guided toward the target throughout the search process by unconscious memory. However, another possibility is a two-state account whereby participants either have memory for the target location and rapidly move towards it, or they have no memory (conscious or unconscious) for the target location and effectively search for the target at random (e.g., Johnson et al., 2007; Smyth \& Shanks, 2008) - and the existence of these two subsets of old trials would not be apparent when examining the average effects. An effect analogous to this two-state account was seen in the first saccade accuracy results above, such that recollection appeared to improve average first 
saccade accuracy by causing a subset of recollected trials to have highly precise first saccades, with the remaining trials appearing to be based on guesses (Fig. 3b). If a similar two-state account were the case for scanpath efficiency, scanpath ratio for the majority of old scenes might be similar to that of the new scenes (i.e., there was no effect of memory for these scenes at all), but there may exist a subset of old scenes for which there was unconscious memory and a very highly efficient scanpath. For these latter trials, the eyes would have moved quickly to the target location, and therefore would be expected to elicit very few saccades and highly efficient scanpaths. To assess these possibilities, we considered two sets of analyses.

First, to quantify scanpath efficiency throughout the search process, we examined delta distance - the extent to which each saccade brought the eyes closer to the target—which allowed us to determine if memory led to consistently elevated efficiency throughout the course of search, for trials eliciting different numbers of saccades. Delta distance was calculated separately for each saccade in the test phase, and was defined as the change in distance from the target from the beginning to the end of the saccade (Table 2). As such, a larger delta distance value indicates that the saccade brought the eyes closer to the target. Given that the effects of memory on scanpath ratio were observed across all memory responses for old scenes, such that previous viewing of a scene improved scanpath ratio irrespective of memory response, all old scenes were included in the model of delta distance. If only a subset of trials involved direct guidance to the target whereas the rest proceeded as random search, as in the two-state account, one would expect delta distance to be greater for old scenes only at low saccade indexes (i.e., shorter trials).

Fig. $4 \mathrm{~b}$ shows that delta distance was greater for old scenes than for new scenes, and this effect was observed across both low and high saccade indexes. That is, there was a significant effect of old/new status of a scene, $t(16400)=3.15, p=.002, d=0.05$, and no interaction between old/new status and saccade index, $t(16461)=-0.37, p=.71$. This indicates that memory enhanced scanpath efficiency similarly for both early and late saccades, and for trials ranging from few to many saccades. Similar results were observed when we restricted the analysis to scenes that were endorsed as "sure new," such that there was a significant effect of old/new status, $t(6527)=2.72, p=.007, d=0.07$, and no old $/$ new by saccade index interaction, $t(6517)=-0.50, p=.62$. Lastly, when analysis was restricted to old scenes, there was no effect of any memory response on delta distance, $\beta=-0.0004$, $t(12370)=0.05, p=.96$, suggesting that improvements in delta distance were uniquely related to unconscious memory, without an influence of familiarity or conscious recollection. Therefore, examination of delta distance suggests that unconscious memory gradually led the eyes to the target throughout the course of search. (For a related analysis that controls for the total number of saccades made in a trial, see Appendix A.)

Second, we examined the histograms of scanpath ratio and number of saccades elicited by old and new scenes in the test phase, to assess whether there was evidence for two different underlying distributions of scanpaths for the old scenes. That is, according to the two-state account, one might expect to see a majority of trials with high scanpath ratios and many saccades, reflecting random search equivalent to the search process observed in new scenes —along with a subset of old trials with scanpath ratios near ceiling (i.e., around 1) and few 
saccades, reflecting that the eyes were guided directly to the target. This two-state account would be corroborated by distributions that are similar to those seen in first saccade accuracy (Fig. 3b), such that a subset of old trials are near ceiling, and the remaining distribution is an evenly reduced version of the new trial distribution. That is, the subset of near-ceiling trials would have appeared to be evenly sampled from the remainder of the distribution rather than resulting from an overall shift of the distribution towards higher performance. However, instead of this kind of mixture distribution, both Fig. $4 \mathrm{c}$ and d suggests that the distribution of old trials is simply shifted left towards lower scanpath ratios and fewer saccades, respectively, than new trials. As such, the distributions do not suggest that there was a mixture of trials with memory leading the eyes directly to the target and trials that are indistinguishable from new trials. Instead, the distributions suggest that there was an increase in search efficiency affecting the majority of trials, in contrast to the effects of recollection on first saccade accuracy.

Replication.-All of the test phase analyses above (throughout Section 3.2) were conducted using the scenes that were presented once in the learning phase, as we expected the scenes that were presented three times to be at ceiling levels of recognition. However, we also examined performance on these latter scenes for the sake of completeness, and the analyses largely replicated the effects we observed in the scenes presented once. All significant and null effects replicated for degree error of first saccade (recollection effect: $t(431)=-3.01, p=.003, d=-0.29)$, scanpath ratio (unconscious effect: $t(241)=-2.48, p=$. $013, d=-0.32)$, and delta distance (old/new effect: $t(4213)=6.86, p<.0001, d=0.21$ ), except for the null interaction between saccade index and old/new status in delta distance, $\beta$ $=-0.03, t(6174)=-3.62, p<.001$. This interaction indicates that the magnitude of the effect of old/new status on delta distance changed over the course of the trial or across trials of different lengths, which could suggest that the influence of unconscious memory on eye movement guidance was restricted to a particular point in the search process or to trials consisting of a certain number of saccades. However, examination of the data revealed that this significant interaction was instead due to a transient reduction in the effect of old/new status on delta distance around the 21st-25th saccade (likely due to having few trials of that length for scenes presented three times), whereas old/new status showed a consistent effect throughout the rest of the saccades. Therefore, analyses using the scenes presented three times provided an internal replication of the main results outlined above.

\section{Discussion}

In the present study, we examined eye movements made during a visual search task and used a subjective report procedure that isolated the contributions of conscious recollection, unconscious memory, and familiarity strength. The present results demonstrate that eye movements made while viewing real-world scenes can be influenced by both conscious and unconscious memory. Whereas conscious recollection for a scene uniquely improved the accuracy of the first eye movement in a search task, unconscious memory uniquely improved participants' search efficiency and gradually guided the eyes towards the target over the course of a trial. Examination of the distributions underlying these effects suggested that conscious recollection may lead to very highly accurate first saccades on a subset of 
trials, whereas unconscious memory may instead lead to a general and diffuse improvement in the efficiency of each saccade on the majority of trials. Furthermore, Bayesian analyses indicated that these memory effects on eye movements may be independent, such that conscious memory did not influence scanpath efficiency, and unconscious memory did not influence first saccade accuracy — and indicated that familiarity strength did not influence either of these patterns of eye movements. These results build upon previous work by introducing a novel paradigm that allows for the separation of conscious and unconscious memory from intermediate strength-based memory that could bias these measures, and, through eye-tracking, allows for the decomposition of previously reported reaction time effects.

By demonstrating that multiple types of memory can influence attention simultaneously, the present results may be able to explain past conflicting findings on whether contextual cueing effects are due to conscious or unconscious memory. That is, the present finding of multiple forms of memory contributing to contextual cueing suggests that the contradictory conclusions reached in prior studies may be due to the dichotomous old/new memory assessments used, and to differences in memorability of the stimuli used. Specifically, many of the studies reporting that contextual cueing is driven only by unconscious memory, due to the absence of conscious memory for the stimuli, used abstract arrays of letters as the background context (e.g., Chun, 2000; Chun \& Jiang, 1998; Goujon, Didierjean, \& Thorpe, 2015). Abstract arrays generally do not elicit levels of conscious memory that are detectable in the dichotomous old/new memory tests that are used in these studies, likely due to very high similarity between stimuli and a lack of semantic information. On the other hand, those studies attributing contextual cueing to conscious memory, due to very high recognition accuracy on dichotomous memory tests, have typically used scenes as stimuli (e.g., Brockmole \& Henderson, 2006a, 2006b) —for which people have exceptionally good memory (Konkle, Brady, Alvarez, \& Oliva, 2010; Standing, 1973). Given that contextual cueing effects are stronger and appear sooner in scenes than in arrays, combined with the present results, it seems likely that contextual cueing in scenes is driven by both conscious and unconscious memory, whereas contextual cueing in abstract arrays of letters may be driven primarily by unconscious memory.

The finding that conscious recollection influenced the first saccade whereas unconscious memory influenced performance throughout the course of search might seem surprising, given that conscious influences of memory are generally thought to be slower than unconscious influences (Schacter, Wagner, \& Buckner, 2000; Yonelinas, 2002). We suspect that one reason that the conscious memory effects occurred so early in search is because participants saw a preview of and made explicit memory judgments about each test phase scene prior to searching for the target, and as such, eye movements related to conscious recollection may have been planned prior to the onset of the target-containing version of the scene. However, while the explicit recognition response likely did impact the ability of recollection to influence the first eye movement, the same effects on the first saccade were already apparent in the learning phase, where there was no scene preview or memory judgment-suggesting that these early eye movements are observed even without a preview or explicit retrieval demands. We interpret the results as indicating that conscious 
recollection can impact eye movements relatively quickly after stimulus onset, even without a preview.

The current results do not speak directly to questions regarding the neural substrates of these effects, but we believe that combining the present subjective report methods with patient studies or neuroimaging methods may be useful in addressing these questions. For example, there is conflicting evidence regarding whether the hippocampus or regions in the surrounding medial temporal lobe are involved in supporting memory-related patterns of eye movements (Chun \& Phelps, 1999; Giesbrecht, Sy, \& Guerin, 2013; Manns \& Squire, 2001; Preston \& Gabrieli, 2008), and the present paradigm may prove useful in informing this debate when combined with measures of neural substrates. Furthermore, a growing body of memory research has indicated that recollection is dependent on the hippocampus (Eichenbaum et al., 2007), and that it is associated with relatively high-resolution information (Yonelinas, 2013). Specifically, the hippocampus has been shown to provide high-resolution spatial information about previous events, whereas cortical regions outside the hippocampus can support less precise spatial information such as the general quadrant in which objects were previously encountered (Koen, Borders, Petzold, \& Yonelinas, 2017; Kolarik et al., 2016). The fact that recollection in the current study was associated with highly accurate first saccades towards the target location is consistent with the proposed role of the hippocampus in supporting high-precision memory responses.

Whereas the deployment of highly precise memory representations supported by the hippocampus is a feasible mechanism through which recollection may influence first saccade accuracy, the potential mechanisms underlying the influence of unconscious memory on scanpath efficiency are less easily identified. There is evidence, however, for relational memory that can be expressed through eye movements in the absence of awareness for that memory (Hannula et al., 2010; Ryan et al., 2000), and it has been proposed that relational memory is instrumental in binding targets to contexts in support of contextual cueing (Chun \& Jiang, 2003). It is therefore possible that the present findings of guidance by unconscious memory may reflect relational memory, which could use contextual scene information to gradually guide the eyes towards the region containing the target. Another possibility is that unconscious memory may improve efficiency throughout the search process by reducing the extent to which saccades are made to incorrect regions. That is, perhaps increased fluency of perceptual processing, resulting from prior exposure (Schacter et al., 2000; Voss \& Paller, 2008), reduces the amount of visual information needed to reject incorrect regions. Studies aimed at identifying the mechanisms underlying the present effects would be useful in determining how unconscious memory influences attention.

In addition to furthering our understanding of how experience guides eye movements, these findings pave the way for a variety of practical applications. For example, extending this work to real-world tasks could allow us to understand and enhance the influence of experience on radiologists' ability to detect cancers in x-ray images, or TSA agents' ability to find weapons in luggage (Wolfe, Brunelli, Rubinstein, \& Horowitz, 2013; Wolfe, 2016): if these results extend to classes of images in addition to repeated instances of an image, perhaps eye movement measures could detect unconscious forms of memory-informed 
search that are not available to conscious report. Moreover, to the extent that eye movements can be used to index different forms of memory, these measures may provide an efficient means to quickly detect different types of memory impairments such as those observed in aging and Alzheimer's disease. Future research is needed to determine whether the presently identified patterns of eye movements may generalize to other conditions, such that they may serve as indicators of conscious and unconscious memory influences.

\section{Acknowledgments}

Funding: This work was supported by the National Eye Institute of the National Institutes of Health under Award Numbers R01EY025999 and R01EY027792. The content is solely the responsibility of the authors and does not necessarily represent the official views of the National Institutes of Health.

\section{Appendix A}

\section{A.1. Statistical analyses}

\section{Correlations between search speed reaction time (RT) and eye movement measures.}

To further verify that the eye movement measures used were reflective of search performance, and therefore contextual cueing performance, we determined whether the eye movement measures were related to RT. An analysis of all of the trials in which the target was identified, throughout both the learning and test phases, showed a significant correlation between scanpath ratio and RT $(r=0.75, p<.0001)$. The correlation between first saccade accuracy and RT was small, but also significant $(r=0.12, p<.0001)$. Despite the weaker relationship between first saccade accuracy and RT (likely driven by the much smaller proportion of total trial time indexed by first saccade accuracy), RT appears to be related to first saccade accuracy independently from improvements in scanpath ratio, as first saccade accuracy remains significant $(p<.0001)$ in predicting RT even when scanpath ratio is controlled for.

\section{Influence of encoding time.}

Due to the possibility of confounds stemming from the expected relation between higher initial viewing time (i.e., RT on first presentation) and stronger memory, we repeated all linear mixed effects models (Table 1 ) assessing the relation between memory and eye movement patterns with the additional covariate of RT for the first presentation of the scene. This was not found to alter the pattern of results. 


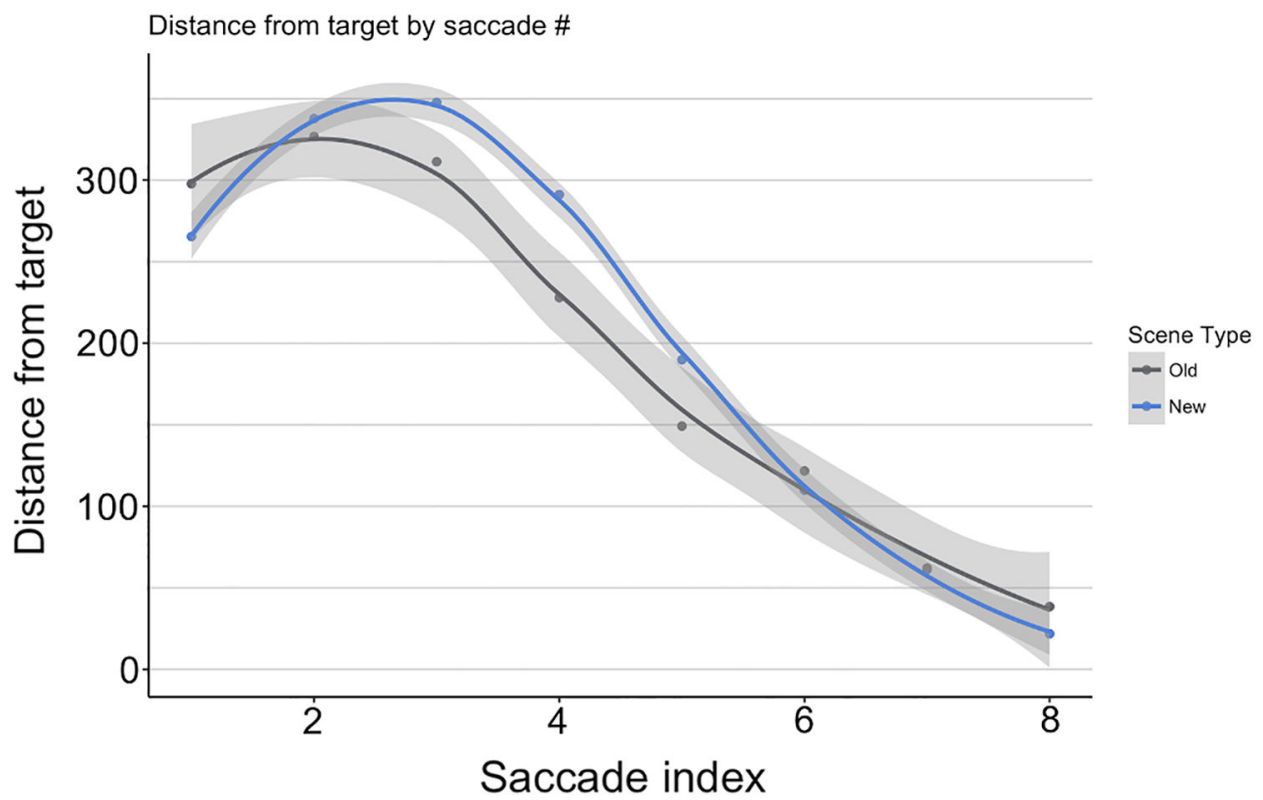

Fig. A.1.

Absolute distance from the target over the course of the trial, for trials in which the target was found within 6-8 saccades. All scenes were endorsed as "sure new," and are sorted by whether or not they appeared in the learning phase. Shaded regions represent the standard error of the mean. The same smoothing method was used as that in Fig. 4b. 


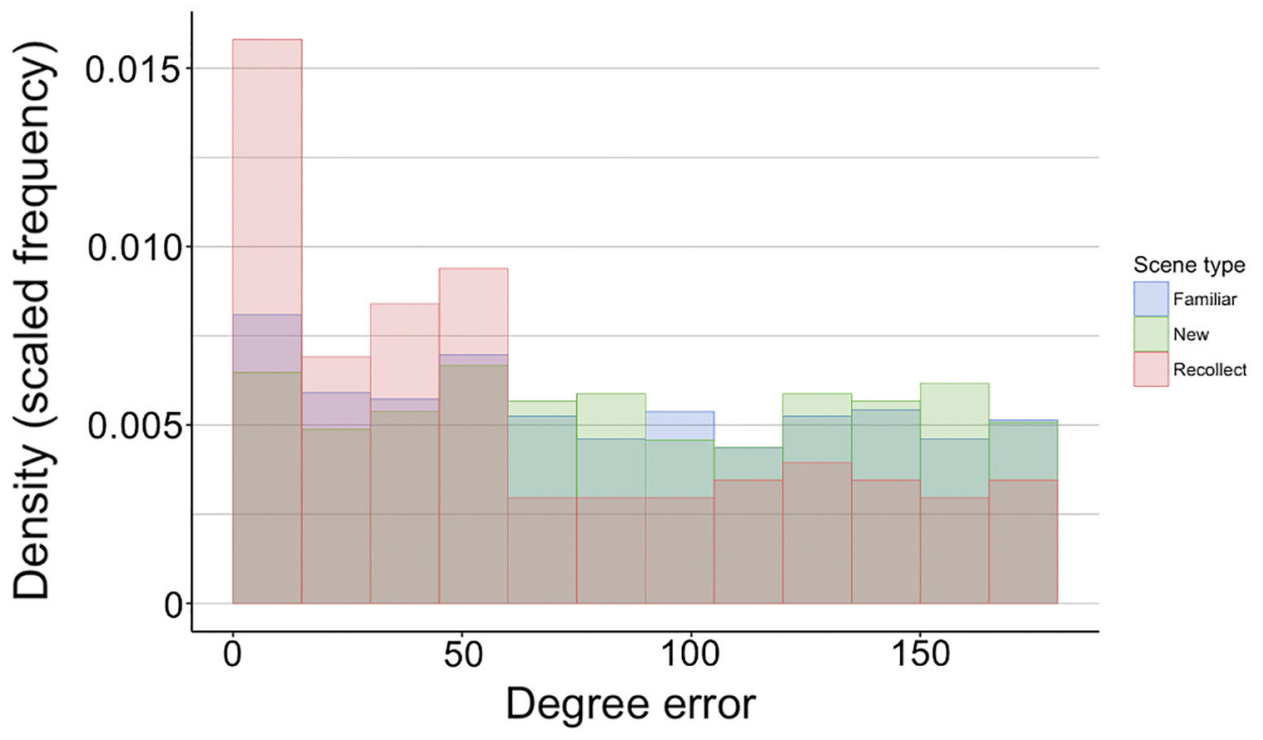

Fig. A.2.

Histogram of first saccade accuracy in the test phase, sorted by scene type. "New" scenes include all new scenes; "Familiar" scenes include old scenes presented once that were not recollected, and "Recollect" scenes include old scenes presented once that were recollected.

\section{Scanpath ratio.}

Scanpath ratio values were skewed; to ensure that skew did not significantly impact the observed effects, we re-ran the analyses with log-transformed scanpath ratio values, and found that the unconscious effect held $(p<.001)$ as did the null effects of familiarity and recollection. The other variables of interest were not skewed.

The first calculation of scanpath ratio presented in the main text (Section 3.2) included data from the first saccade. To provide statistical separation of scanpath ratio from first saccade accuracy, models of the effect of memory on this measure of scanpath ratio included a covariate of first saccade accuracy. That is, Fig. 4a and the statistics reported in the main text assessed the influence of memory on scanpath ratio for a given degree of first saccade accuracy. The null effect of recollection on scanpath ratio was similar regardless of whether or not this covariate was included, however (with covariate, $p=.14$, without covariate: $p=$. 12). Furthermore, inclusion of the first saccade accuracy covariate did not alter the pattern of results with respect to familiarity nor unconscious memory. This covariate was not included in the models assessing scanpath ratio that was calculated from the second saccade onward.

\section{Decomposition of scanpath efficiency: additional analysis.}

Delta distance was computed to determine whether scanpath efficiency was elevated throughout the course of the search process, and for trials consisting of different numbers of saccades. In order to have a sufficient number of trials in each saccade index bin, trials consisting of 30 or fewer saccades were used in this analysis. However, because different trials required different numbers of saccades to locate the target, plotting all of these trials together may obscure effects that vary depending on trial length. To observe the trends in 
unconscious memory guidance throughout trials consisting of a similar number of saccades, we plotted absolute distance from the target, over the course of saccades, for trials in which the target was found within 6-8 saccades (Fig. A.1). (The mode number of saccades in a trial was 7; Fig. 4d) This plot corroborates the conclusion that unconscious memory gradually guided the eyes towards the target over the course of the trial, as the eyes appear to be consistently closer to the target in old scenes.

\section{Plotting.}

Figs. 2, 3a and 4a were plotted using least-squares means obtained using the linear mixed effects models. Thus, the plots control for participant and image as in the models reported in the text-but the trends are similar when the raw data is plotted instead. Error bars in these figures were calculated using the standard error values generated by the lsmeans package in R (Lenth, 2016).

Fig. $3 b$ presents a density plot of first saccade accuracies, but a standard version of the histogram is presented below (Fig. A.2). Proportions (i.e., scaled frequency) are used to facilitate comparison between distributions containing different numbers of trials.

\section{Degrees of freedom.}

Because the degrees of freedom were derived from the linear mixed effects models using the Satterthwaite approximation, the degrees of freedom were based on the number of trials included in an analysis rather than the number of participants included in the experiment. Therefore, a high value for degrees of freedom reflects the fact that a large number of data points were included in a given model. Degrees of freedom obtained using this method often contain decimals, but they were rounded to the nearest integer in the manuscript.

\section{Appendix B.: Supplementary material}

Supplementary data to this article can be found online at https://doi.org/10.1016/j.cognition. 2019.01.007.

\section{References}

Althoff RR, \& Cohen NJ (1999). Eye-movement-based memory effect: A reprocessing effect in face perception. Journal of Experimental Psychology: Learning Memory and Cognition, 25(4), 9971010. 10.1037/0278-7393.25.4.997.

Brockmole JR, \& Henderson J (2006a). Recognition and attention guidance during contextual cueing in real-world scenes: Evidence from eye movements. Quarterly Journal of Experimental Psychology, 59(7), 1177-1187. 10.1080/17470210600665996.

Brockmole JR, \& Henderson JM (2006b). Using real-world scenes as contextual cues for search. Visual Cognition, 13(1), 99-108. 10.1080/13506280500165188.

Castelhano MS, \& Henderson JM (2007). Initial scene representations facilitate eye movement guidance in visual search. The Journal of Experimental Psychology: Human Perception and Performance. 10.1037/0096-1523.33.4.753.

Chun MM (2000). Contextual cueing of visual attention. Trends in Cognitive Sciences. 10.1016/ S1364-6613(00)01476-5. 
Chun MM, \& Jiang Y (1998). Contextual cueing: implicit learning and memory of visual context guides spatial attention. Retrieved from Cognitive Psychology, 36, 28-71. http:// resolver.scholarsportal.info/resolve/00100285/v36i0001/28_ccilamovcgsa.xml. [PubMed: 9679076]

Chun MM, \& Jiang Y (2003). Implicit, long-term spatial contextual memory. Journal ofExperimental Psychology: Learning, Memory, and Cognition, 29(2), 224.

Chun MM, \& Phelps EA (1999). Memory deficits for implicit contextual information in amnesic subjects with hippocampal damage. Nature Neuroscience, 2, 844-847. 10.1038/12222. [PubMed: 10461225]

Donaldson W (1996). The role of decision processes in remembering and knowingMemory \& Cognition, Vol, 24).

Eichenbaum H, Yonelinas AP, \& Ranganath C (2007). The medial temporal lobe and recognition memory. Annual Review of Neuroscience, 30, 123-152. 10.1146/annurev.neuro. 30.051606 .094328 .

Giesbrecht B, Sy JL, \& Guerin SA (2013). Both memory and attention systems contribute to visual search for targets cued by implicitly learned context. Vision Research, 85, 80-89. 10.1016/j.visres. 2012.10.006. [PubMed: 23099047]

Goujon A, Didierjean A, \& Marmèche E (2007). Contextual cueing based on specific and categorical properties of the environment. Visual Cognition, 15(3), 257-275. 10.1080/13506280600677744.

Goujon A, Didierjean A, \& Thorpe S (2015). Investigating implicit statistical learning mechanisms through contextual cueing. Trends in Cognitive Sciences, 19(9), 524-533. 10.1016/j.tics. 2015.07.009. [PubMed: 26255970]

Hannula DE (2010). Worth a glance: Using eye movements to investigate the cognitive neuroscience of memory. Frontiers in Human Neuroscience, 4, 166 10.3389/fnhum.2010.00166. [PubMed: 21151363]

Hannula DE, Baym CL, Warren DE, \& Cohen NJ (2012). The eyes know: Eye movements as a veridical index of memory. Psychological Science, 23(3), 278-287. 10.1177/0956797611429799. [PubMed: 22327015]

Hannula DE, Ranganath C, Ramsay IS, Solomon M, Yoon J, Niendam TA, ... Ragland JD (2010). Use of eye movement monitoring to examine item and relational memory in schizophrenia. Biological Psychiatry, 68(7), 610-616. 10.1016/j.biopsych.2010.06.001. [PubMed: 20673874]

Henderson JM, \& Hollingworth A (2003). Eye movements and visual memory:Detecting changes to saccade targets in scenes. Perception and Psychophysics, 65(1), 58-71. 10.3758/BF03194783. [PubMed: 12699309]

Hollingworth A, Williams CC, \& Henderson JM (2001). To see and remember: Visually specific information is retained in memory from previously attended objects in natural scenes. Psychonomic Bulletin and Review, 8(4), 761-768. 10.3758/BF03196215. [PubMed: 11848597]

Jeffreys H (1961). Theory of probability (3rd ed.). Oxford, England: Clarendon.

Johnson JS, Woodman GF, Braun EL, \& Luck SJ (2007). Implicit memory influences the allocation of attention in visual cortex. Psychonomic Bulletin \& Review, 14(5), 834-839. 10.3758/BF03194108. [PubMed: 18087946]

Kafkas A, \& Montaldi D (2011). Recognition memory strength is predicted by pupillary responses at encoding while fixation patterns distinguish recollection from familiarity. The Quarterly Journal of Experimental Psychology, 64(10), 1971-1989. 10.1080/17470218.2011.588335. [PubMed: 21838656]

Kafkas A, \& Montaldi D (2012). Familiarity and recollection produce distinct eye movement, pupil and medial temporal lobe responses when memory strength is matched. Neuropsychologia, 50(13), 3080-3093. 10.1016/j.neuropsychologia.2012.08.001. [PubMed: 22902538]

Koen JD, Borders AA, Petzold MT, \& Yonelinas AP (2017). Visual short-term memory for high resolution associations is impaired in patients with medial temporal lobe damage. Hippocampus, 27, 184-193. 10.1002/hipo.22682. [PubMed: 27859914]

Kolarik BS, Shahlaie K, Hassan A, Borders AA, Kaufman KC, Gurkoff G, ... Ekstrom AD (2016). Impairments in precision, rather than spatial strategy, characterize performance on the virtual Morris Water Maze: A case study. Neuropsychologia, 80, 90-101. 10.1016/j.neuropsychologia. 2015.11.013. [PubMed: 26593960] 
Konkle T, Brady TF, Alvarez GA, \& Oliva A (2010). Scene memory is more detailed than you think: The role of categories in visual long-term memory. Psychological Science, 21(11), 1551-1556. 10.1177/0956797610385359. [PubMed: 20921574]

Kuznetsova A, Brockhoff PB, \& Christensen RHB (2017). lmerTest package: Tests in linear mixed effects models. Journal of Statistical Software, 82(13), 1-19. 10.18637/jss.v082.i13.

Lenth RV (2016). Least-squares means: The R package lsmeans. Journal of StatisticalSoftware, 69(1)10.18637/jss.v069.i01.

Manns JR, \& Squire LR (2001). Perceptual learning, awareness, and the hippocampus. Hippocampus, 11(6), 776-782. 10.1002/hipo.1093. [PubMed: 11811672]

Peterson MS, \& Kramer AF (2001). Attentional guidance of the eyes by contextual information and abrupt onsets. Perception \& Psychophysics, 63(7), 1239-1249. 10.3758/BF03194537. [PubMed: 11766947]

Preston AR, \& Gabrieli JDE (2008). Dissociation between explicit memory and configural memory in the human medial temporal lobe. Cerebral Cortex, 18, 2192-2207. 10.1093/cercor/bhm245. [PubMed: 18234685]

Rosenthal R, \& Rosnow RL (1991). Essentials of behavioral research: Methods and data analysis McGraw-Hill series in psychology. Retrieved from https://lggy89ten01.storage.googleapis.com/ MDA3MzUzMTk2MA==01.pdf.

Ryan JD, Althoff RR, Whitlow S, \& Cohen NJ (2000). Amnesia is a deficit in relational memory. Psychological Science, 11(6), 454-461. 10.1111/1467-9280.00288. [PubMed: 11202489]

Ryan JD, Hannula DE, \& Cohen NJ (2007). The obligatory effects of memory on eye movements. Memory, 15(5), 508-525. 10.1080/09658210701391022. [PubMed: 17613794]

Schacter DL, Wagner AD, \& Buckner RL (2000). Memory systems of 1999 The oxford handbook of memory (pp. 627-643).

Schlagbauer B, Muller HJ, Zehetleitner M, \& Geyer T (2012). Awareness in contextual cueing of visual search as measured with concurrent access- and phenomenal-consciousness tasks. Journal of Vision, 12(11), 1-12. 10.1167/12.11.25.

Sharot T, Davidson ML, Carson MM, \& Phelps EA (2008). Eye movements predict recollective experience. PLoS ONE, 3(8), 10.1371/journal.pone.0002884.

Smith CN, Hopkins RO, \& Squire LR (2006). Experience-dependent eye movements, awareness, and hippocampus-dependent memory. The Journal of Neuroscience: The Official Journal of the Society for Neuroscience, 26(44), 11304-11312. 10.1523/JNEUROSCI.3071-06.2006. [PubMed: 17079658]

Smith CN, \& Squire LR (2008). Experience-dependent eye movements reflect hippocampusdependent (Aware) memory. Journal of Neuroscience, 28(48), 12825-12833. 10.1523/ JNEUROSCI.4542-08.2008. [PubMed: 19036976]

Smith CN, \& Squire LR (2017). When eye movements express memory for old and new scenes in the absence of awareness and independent of hippocampus. Learning \& Memory, 24(2), 95-103. 10.1101/lm.043851.116. [PubMed: 28096499]

Smyth AC, \& Shanks DR (2008). Awareness in contextual cuing with extended and concurrent explicit tests. Memory and Cognition, 36(2), 403-415. 10.3758/MC.36.2.403. [PubMed: 18426069]

Standing L (1973). Learning 10,000 pictures. Quarterly Journal of Experimental Psychology, 25(2), 207-222. 10.1080/14640747308400340. [PubMed: 4515818]

Voss JL, \& Paller KA (2008). Brain substrates of implicit and explicit memory: The importance of concurrently acquired neural signals of both memory types. Neuropsychologia, 46(13), 30213029. 10.1016/j.neuropsychologia.2008.07.010. [PubMed: 18691605]

Wolfe JM (2016). Use-inspired basic research in medical image perception. s(pp. 1-17).. 10.1186/ s41235-016-0019-2.

Wolfe JM, Brunelli DN, Rubinstein J, \& Horowitz TS (2013). Prevalence effects in newly trained airport checkpoint screeners: Trained observers miss rare targets, too. Journal of Vision, 13(3), 19. 10.1167/13.3.33.

Yonelinas AP (2002). The nature of recollection and familiarity: A review of 30 years of research. Journal of Memory and Language, 46, 441-517. 10.1006/jmla.2002.2864. 
Yonelinas AP (2013). The hippocampus supports high-resolution binding in the service of perception, working memory and long-term memory. Behavioural Brain Research, 254, 34-44. 10.1016/j.bbr. 2013.05.030. [PubMed: 23721964]

Zhang W, \& Luck SJ (2008). Discrete fixed-resolution representations in visual working memory. Nature, 453(7192), 233-235. 10.1038/nature06860. [PubMed: 18385672] 
(A)

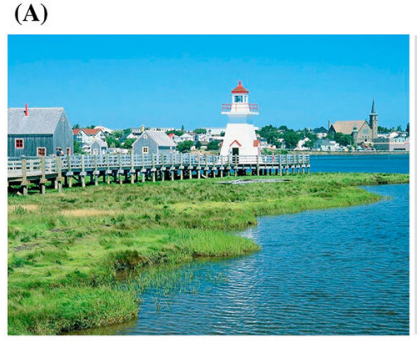

(B)

Is this photo old or new?

(4) Maybe it's old

(3) I don't know

2 Maybe it's new

(1) I'm sure it's new
(C)

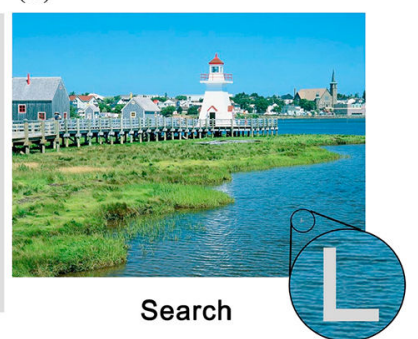

Fig. 1.

Test phase trial. In each test phase trial, participants saw a preview of a scene without its target, then reported whether they remembered viewing the scene in the learning phase. They subsequently searched the scene again as they did in the learning phase. (A) The preview of the scene, which did not contain the target letter. (B) A schematic of the recognition judgment probe; participants were asked to rate their memory for the scenes on a 1-6 scale capturing varying levels of confidence. (Note that colors and spacing for "Recollect old" and "I'm sure it's new" responses are used for the sake of illustration; they were not visually unique from the other responses in the actual memory probe.) (C) The scene presented again for the subsequent search task; a zoomed in view of the target letter ("L") is provided for visualization purposes. (For interpretation of the references to colour in this figure legend, the reader is referred to the web version of this article.) 
(A)

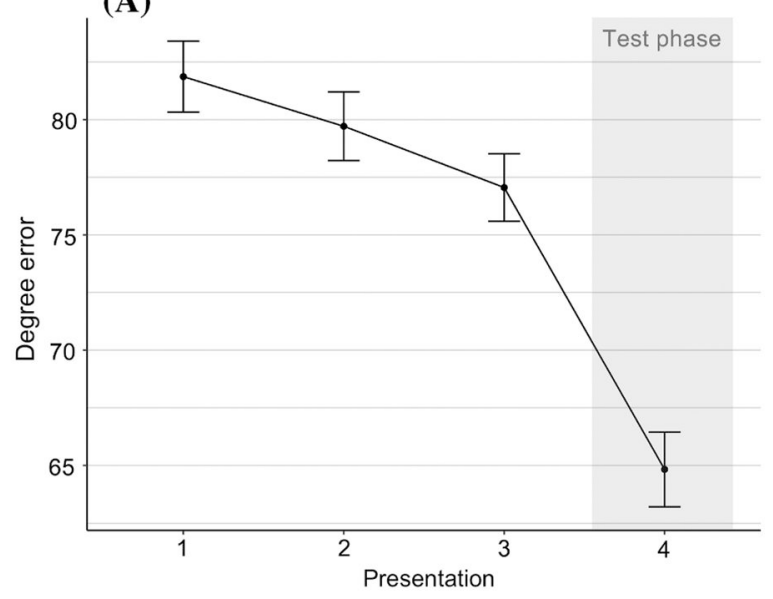

(B)

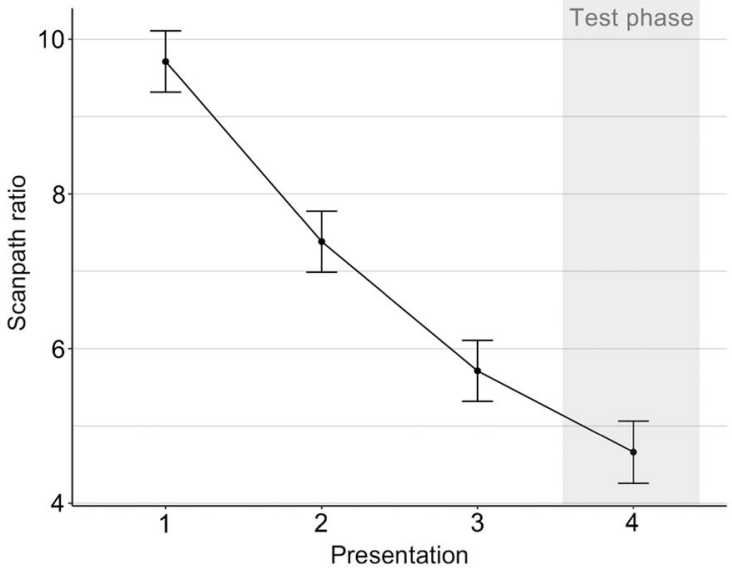

Fig. 2.

Improvement in eye movement measures for scenes presented three times in the learning phase, over the course of the learning phase (presentations 1-3) and the test phase (presentation 4). Least-squares means controlling for participant and image are plotted, and the error bars represent the standard error of these estimated means from the model. (A) First saccade accuracy. (B) Scanpath efficiency. 
(A)

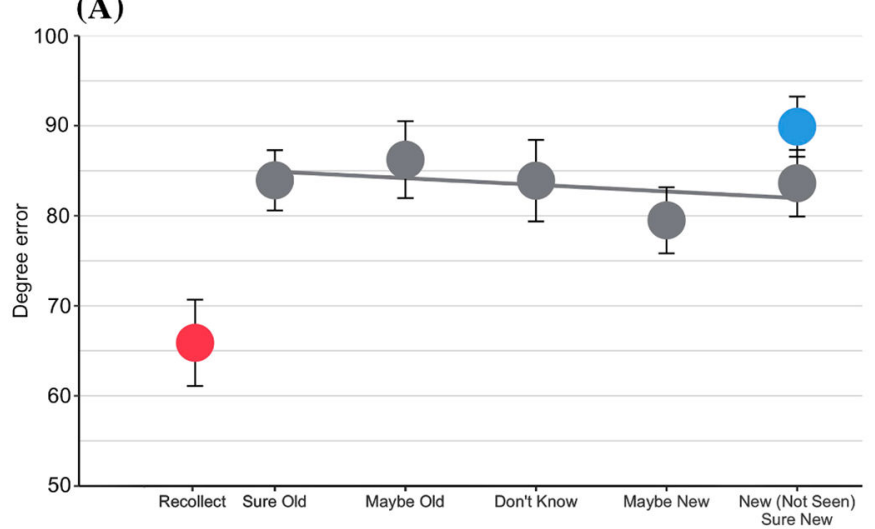

(B)

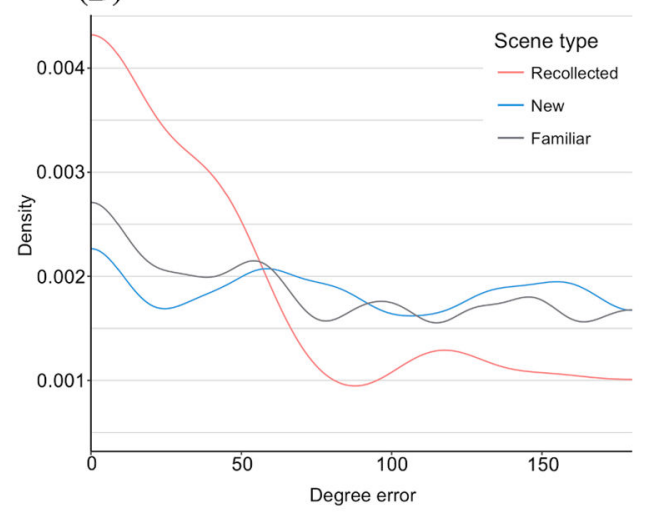

Fig. 3.

(A) Test phase average first saccade accuracy (the angular degree error between the first saccade's direction from the center, and the direction of the target from the center; Table 2) by memory response. The "New (Not Seen)" (blue) scenes were correct rejections (i.e., "sure new" responses) of new scenes, which are included as a memory-less control; all other data points were old scenes, and these responses therefore include hits (recollect, sure old, and maybe old) and misses (don't know, maybe new, sure new). Least-squares means controlling for participant and image are plotted, and the error bars represent the standard error of these estimated means from the model. The gray line represents the regression line across the familiarity-based responses used in the analysis of memory strength effects. (B) Density plot (i.e., smoothed histogram of scaled frequencies) showing the distribution of first saccade accuracy by memory type. (B)The curves were generated based on the histogram of first saccade accuracies using a Gaussian smoothing kernel, and bin widths were set to $1 / 3$ of the default for the kernel (as determined by the stat_density function within the ggplot2 package in R). The standard histogram, also using scaled frequency to facilitate comparison between categories with different numbers of trials, is plotted in Fig. A.2. (For interpretation of the references to colour in this figure legend, the reader is referred to the web version of this article.) 
(A)

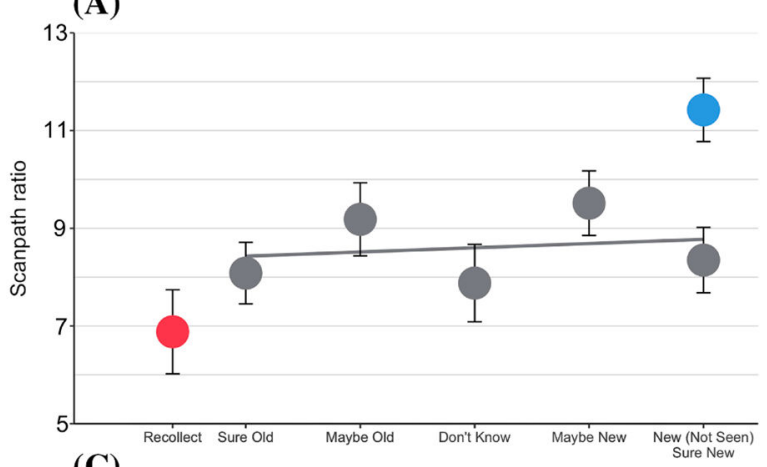

(C)

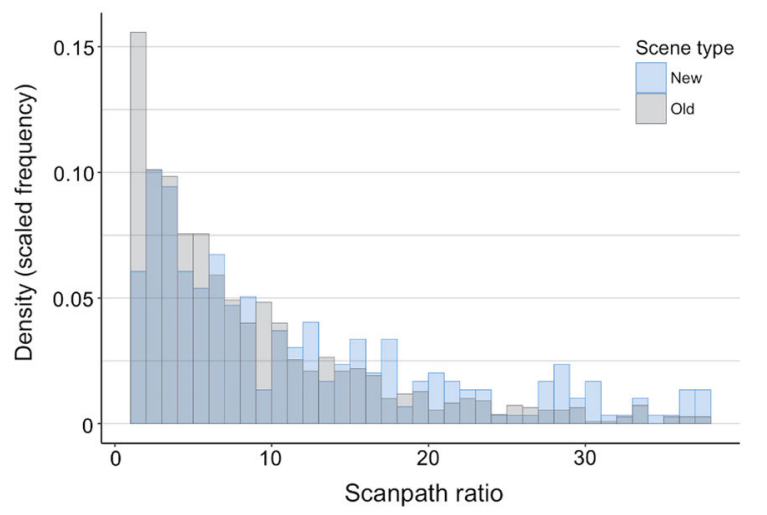

(B)

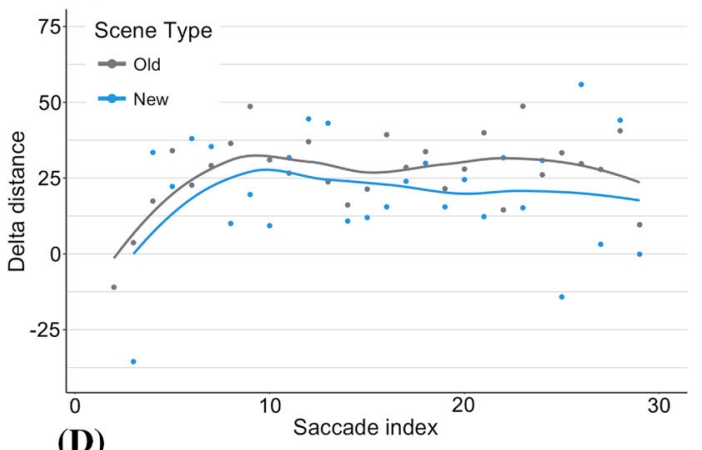

(D)

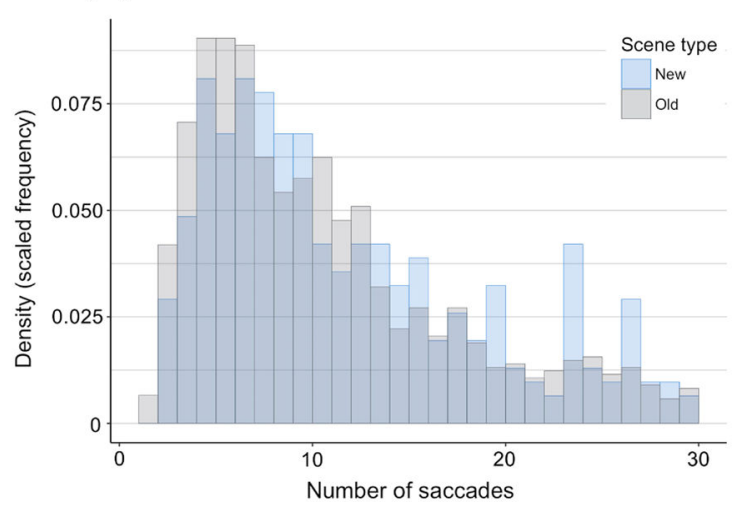

Fig. 4.

(A) Test phase average scanpath ratio (measured as the ratio of the observed scanpath to the shortest path to the target; Table 2) by memory response. The "New (Not Seen)" (blue) scenes were correct rejections (i.e., "sure new" responses) of new scenes, which are included as a memory-less control; all other data points were old scenes, and these responses therefore include hits (recollect, sure old, and maybe old) and misses (don't know, maybe new, sure new). Least-squares means controlling for participant and image are plotted, and the error bars represent the standard error of these estimated means from the model. The gray line represents the regression line across the familiarity-based responses used in the analysis of memory strength effects. (B) Delta distance, measured as the extent to which each saccade brought the eyes closer to the target, over the course of the trial (Table 2). The $\mathrm{x}$-axis represents the saccade index, which is the ordinal saccade number in a trial. Each data point represents the average change in distance from the target (in pixels) for a given saccade index, with a separate data point for each condition (old versus new scenes). (B) The lines were generated using a loess smoothing (i.e., locally weighted smoothing) function in the ggplot2 package in $\mathrm{R}$, which plots local regressions to aid the eye in seeing trends from scatterplots that may not necessarily be best captured by a standard linear regression. (C) Histogram of scanpath ratio values for old and new scenes. (D) Histogram of the number of saccades made in a trial for old and new scenes. Scaled frequency values are presented in both (C) and (D) to facilitate comparisons between the old and new distributions, given that there were different numbers of old and new scenes. In each histogram, the bin width is one unit. Plots (B)-(D) include new scenes given a response of "sure new" (corresponding to the 
blue point in (A)), and all old scenes. (For interpretation of the references to colour in this figure legend, the reader is referred to the web version of this article.) 


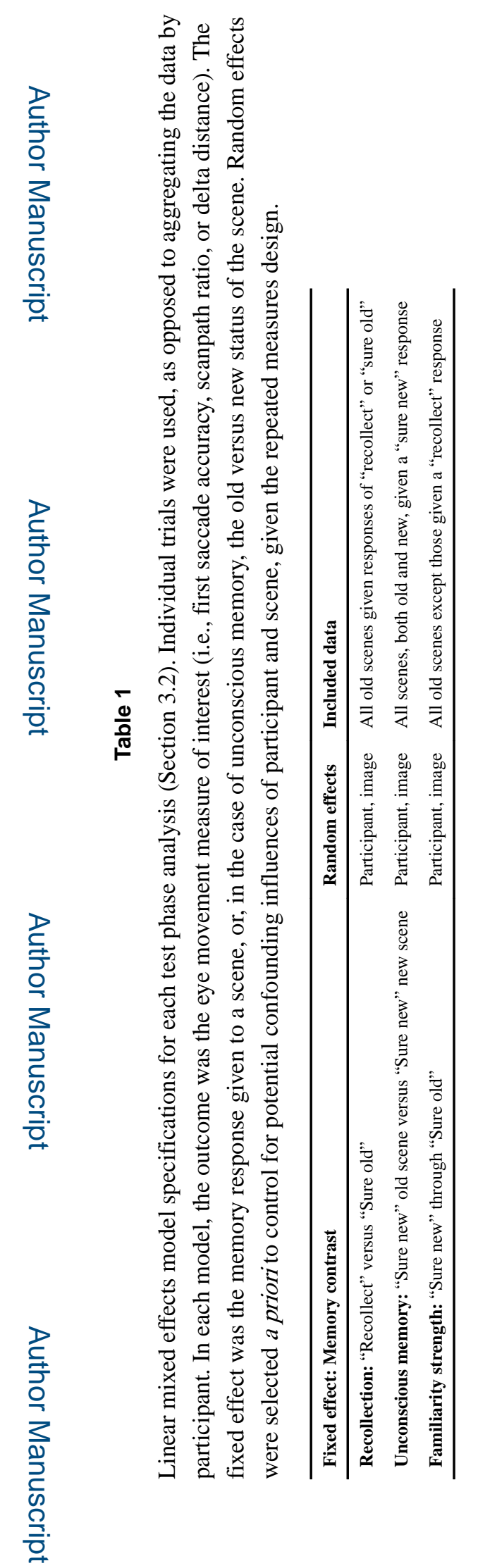

Cognition. Author manuscript; available in PMC 2020 January 06. 


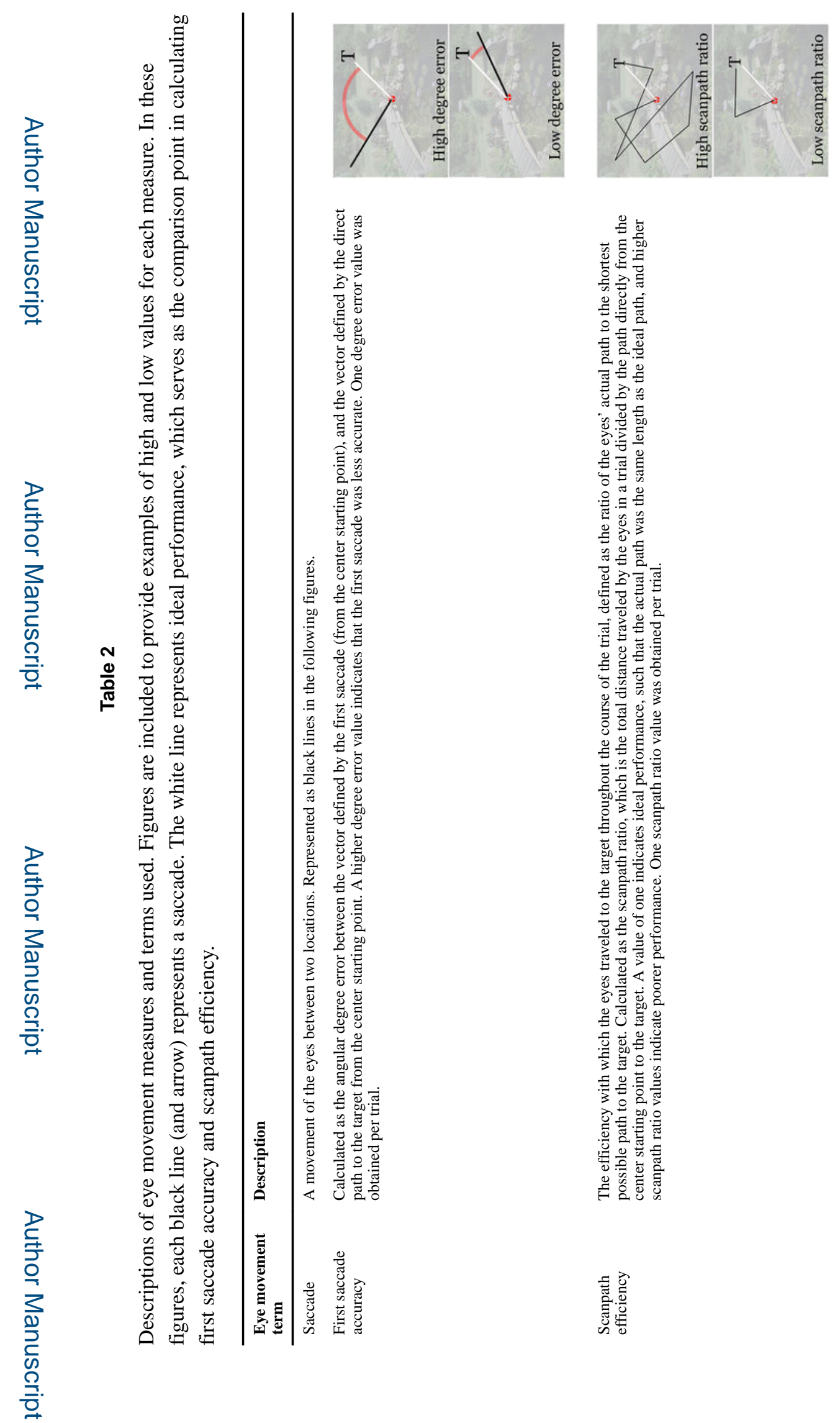

Cognition. Author manuscript; available in PMC 2020 January 06. 


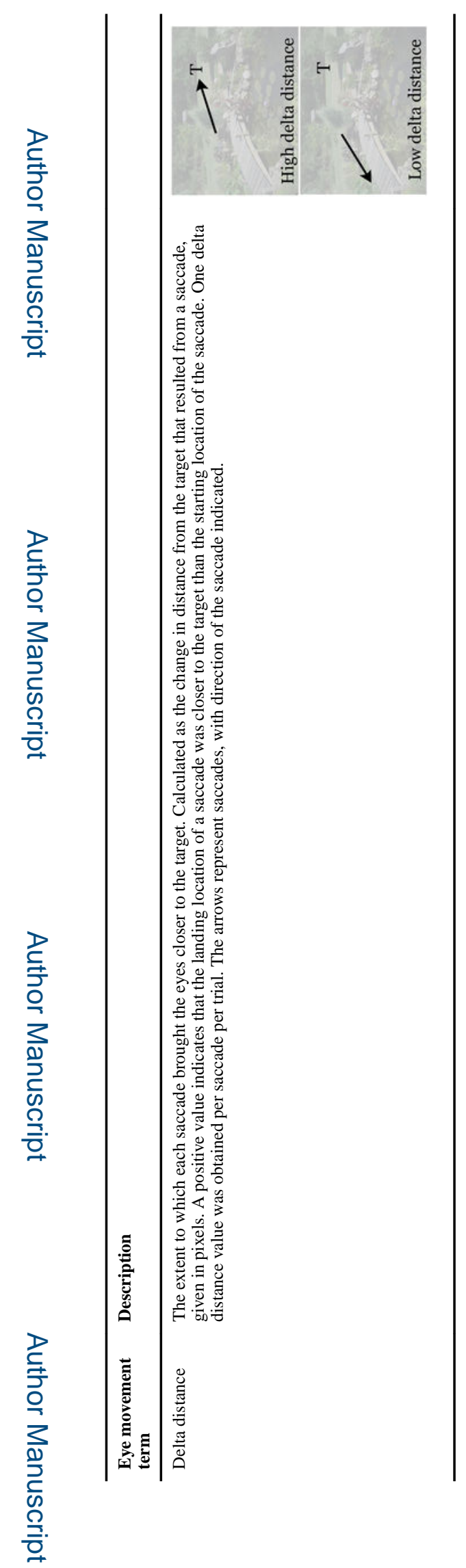

Cognition. Author manuscript; available in PMC 2020 January 06. 